\title{
Expression of Fractalkine Receptor CX3CR1 on Cochlear Macrophages Influences Survival of Hair Cells Following Ototoxic Injury
}

\author{
Eisuke Sato, ${ }^{1}$ H. Elizabeth Shick, ${ }^{2}$ Richard M. Ransohoff, ${ }^{3}$ and Keiko Hirose ${ }^{4}$ \\ ${ }^{1}$ Department of Otolaryngology, Nagoya University, Nagoya, Japan \\ ${ }^{2}$ Lerner Research Institute, Cleveland Clinic, Cleveland, OH, USA \\ ${ }^{3}$ Department of Neurology, Mellen Center, Cleveland Clinic, OH, USA \\ ${ }^{4}$ Department of Otolaryngology, Washington University, 660 South Euclid Avenue, Campus Box 8115, St. Louis, MO 63110, \\ USA
}

Received: 30 July 2009; Accepted: 2 November 2009; Online publication: 21 November 2009

\begin{abstract}
The role of innate immunity and macrophage recruitment to the inner ear after hair cell injury is a subject where little is known. In this paper, we demonstrate recruitment of monocytes and macrophages to the inner ear after kanamycin. We also examined the effect of fractalkine receptor (CX3CR1) deletion in kanamycin ototoxicity. We observed more functional and structural damage in CX3CR1 null mice compared to wild-type and heterozygous littermates. In order to determine if increased susceptibility to kanamycin resulted from CX3CR1 deletion from cochlear leukocytes, we created bone marrow chimeras by transplanting CX3CR1-null bone marrow into wild-type mice whose native bone marrow was ablated by lethal irradiation. These mice were then treated with kanamycin sulfate. Auditory brainstem responses (ABR), hair cell counts, and numbers of macrophages recruited to the cochlea were recorded in irradiated mice that received either wild-type, CX3CR1 heterozygous, or CX3CR1 knockout bone marrow. A strong correlation was present between numbers of macrophages and hair cell death in recipients transplanted with CX3CR1 null marrow. No correlation between macrophage number and hair cell loss was present in mice transplanted with wild-type or CX3CR1 heterozygous marrow. We suggest that CX3CR1 plays a role in modulating the
\end{abstract}

Correspondence to: Keiko Hirose · Department of Otolaryngology · Washington University - 660 South Euclid Avenue, Campus Box 8115, St. Louis, MO 63110, USA. Telephone: +1-314-4544033; fax: +1-314-4542174; email: hirosek@ent.wustl.edu detrimental effects of cochlear macrophages after kanamycin ototoxicity. Our data point to the possibility that CX3CR1-deficient cochlear macrophages exacerbate kanamycin ototoxicity while CX3CR1expressing monocytes do not.

Keywords: CX3CR1, aminoglycoside, ototoxicity, kanamycin, macrophage, B6.CAST-Cdh $23^{\text {ahl+ }}$ mice, bone marrow chimeras

\section{INTRODUCTION}

Aminoglycoside ototoxicity is a well-established cause of sensorineural hearing loss. While many studies have pursued causes of hair cell degeneration after ototoxicity, the events that lead to repair remain under investigation. Degeneration of outer hair cells is the primary cause of hearing loss induced by aminoglycoside antibiotics (Browning et al. 1982). Inflammatory cells have been identified in the avian inner ear and the mammalian cochlea, but it remains unclear whether the inflammatory cell population plays a role in repair or whether it propagates damage and participates in cellular injury after ototoxicity (Warchol 1997, 1999; Bhave et al. 1998; Hirose et al. 2005; Ladrech et al. 2007; Sato et al. 2008).

Prior studies have demonstrated an influx of inflammatory cells into the cochlea after acoustic trauma (Fredelius and Rask-Andersen 1990; Hirose et al. 2005). This cell population can be identified by common leukocyte antigen, CD45, and other markers of the monocyte/macrophage lineage including fractalkine receptor, CX3CR1. CX3CR1 is expressed on monocytes, tissue macrophages, NK cells, activated T 
cells, and microglia. Its ligand, fractalkine, is a membrane-bound glycoprotein expressed on neurons and endothelial cells and acts as a potent adhesion molecule. Fractalkine is also found in a soluble form and has been shown to modulate activated microglia in the central nervous system (Limatola et al. 2005b; Cardona et al. 2006; Lauro et al. 2008).

Our main objective was to investigate the effect of CX3CR1 in the inner ear after ototoxic injury. We addressed, first, how loss of CX3CR1 expression affects outcome after ototoxicity and second, whether leukocytes attracted to the cochlea may be responsible for the observed effect of CX3CR1. In our first experiment, we used CX3CR1 wild-type, heterozygous, and knockout mice and exposed them to systemic kanamycin. We measured three primary outcomes: hearing thresholds, number of cochlear macrophages, and number of surviving hair cells 4 weeks after initial treatment. In this experiment, we found that there was indeed an inflammatory response primarily in the spiral ligament after aminoglycoside exposure that was more pronounced in the CX3CR1 knockout mouse than in wild-type or heterozygous littermates. We also discovered that CX3CR1 deletion resulted in exacerbation of hearing loss caused by kanamycin and in a significant increase in the number of hair cells that were destroyed from aminoglycoside exposure.

Our observations suggest that CX3CR1 is important in attenuating ototoxicity, likely through its expression in migrating leukocytes. In order to isolate the effect of CX3CR1 to hematopoietic cells, we created bone marrow chimeras using donor bone marrow from CX3CR1 knockout mice transplanted into wild-type mice whose bone marrow had been ablated by radiation. Use of bone marrow chimeras obviated the potential confounding effects of CX3CR1 in development or extra hematopoietic expression of CX3CR1. Interestingly, we found a direct correlation between numbers of recruited macrophages and hair cell damage in mice transplanted with CX3CR1 knockout marrow, but not in mice transplanted with CX3CR1 wild-type or heterozygous marrow. Thus, we surmise that cochlear inflammatory cells lack tonic inhibition and exhibit a toxic phenotype when CX3CR1 is absent. Our data suggest a protective role of CX3CR1 mediated by cochlear macrophages, which may limit hearing impairment in aminoglycoside ototoxicity.

\section{MATERIALS AND METHODS}

Experimental animals

Wild-type C57BL6 (B6) and B6.CAST-Cdh23 ${ }^{\text {ahl+ }}$ (B6. CAST) mice were derived from breeders purchased from Jackson Laboratories (Bar Harbor, ME, USA). CX3CR1 knockout mice were kindly provided by Dr. Dan Littman, New York University Medical Center. A targeted deletion of CX3CR1 and replacement with the gene encoding green fluorescent protein rendered all monocytes, macrophages, NK cells, activated $\mathrm{T}$ cells, and microglia endogenously fluorescent in these mice (Jung et al. 2000). Heterozygous mice with one copy of CX3CR1 retain receptor function, whereas homozygous mice have no functional CX3CR1. All animal protocols described were approved by the Institute for Animal Care and Use Committee at Cleveland Clinic.

\section{Generation of bone marrow chimeras}

Embryonic livers from CX3CR1 knockout, CX3CR1 heterozygous, and wild-type mice were harvested as a source of hematopoietic precursor cells as follows: breeder pairs were set up for timed pregnancies, and at E14, pregnant dams were euthanized with an overdose of inhaled isoflurane. The uterus with embryos was surgically removed. Embryos were separated and placed in sterile cold phosphate-buffered saline (PBS) in a laminar flow hood. Fetal livers were dissected from the embryos and pooled in an Eppendorf tube containing $1 \mathrm{ml}$ of sterile PBS. Cell suspensions were made by drawing cells gently through an 18-gauge needle, centrifuged (300 g, $5 \mathrm{~min}$ ), and resuspended in sterile PBS at the final concentration of 20 to 40 million cells per cubic centimeter. Eight-week old wild-type B6.CAST mice were lethally irradiated with a single dose of $980 \mathrm{cGy}$ using Cesium ${ }^{137}$ source (Shepherd Irradiator, JL Shepherd and Associates, San Fernando, CA, USA). Three hours after irradiation, two to four million hematopoietic cells in $100 \mu \mathrm{l}$ sterile saline were infused via the retro-orbital venous sinus. Mice were transplanted with CX3CR1 knockout, heterozygous, or wild-type hematopoietic cells. Mice were weighed daily and examined twice daily for the initial 2 weeks after irradiation.

\section{Drug administration}

Kanamycin sulfate (USB Corporation, Cleveland, $\mathrm{OH}$, USA) was prepared in saline at a concentration of $45 \mathrm{mg} / \mathrm{ml}$. For CX3CR1 wild-type, heterozygous, and knockout mice, a dose of $900 \mathrm{mg} / \mathrm{kg}$ body weight kanamycin sulfate was given twice daily for 15 consecutive days by intraperitoneal (IP) injection. (This is equivalent to $747 \mathrm{mg} / \mathrm{kg}$ kanamycin base). This protocol was adapted from Wu et al. (2001). Control mice for all three genotypes (WT, HET, and KO) were injected twice daily with saline for 15 consecutive days. 
To optimize the dose of kanamycin for bone marrow chimeras in the B6.CAST line, the following preliminary experiments were performed. A doseresponse experiment was conducted using doses of 700,900 , and $1,100 \mathrm{mg} / \mathrm{kg}$ kanamycin sulfate given twice daily for 15 consecutive days by IP injection in wild-type B6.CAST mice (these doses are equivalent to 581, 747, and $913 \mathrm{mg} / \mathrm{kg}$ kanamycin base). We also performed a similar dose-response curve in irradiated B6.CAST mice that had received bone marrow transplant from wild-type B6.CAST mice to determine the effect of radiation on threshold elevation in ototoxicity. We assessed hearing 2 weeks after the last dose of kanamycin and determined that a dose of $800 \mathrm{mg} / \mathrm{kg}$ of kanamycin sulfate $(664 \mathrm{mg} / \mathrm{kg}$ kanamycin base) provided the desired threshold changes. Subsequently bone marrow chimeras in B6.CAST received $800 \mathrm{mg} / \mathrm{kg}$ body weight kanamycin twice daily for 15 consecutive days by IP injection.

\section{Auditory brainstem response (ABR)}

Two weeks after the final antibiotic dose, auditory brainstem response (ABR) was performed on all subjects. Mice were anesthetized with IP injection of xylazine $(20 \mathrm{mg} / \mathrm{kg}$ ) and ketamine (100 mg/kg) before ABR measurement. ABRs were evoked through tone pips and recorded via subcutaneous electrodes placed in the ipsilateral pinna and vertex, with the ground electrode placed in the back near the tail. The stimuli were 5-ms tone pips (0.5-ms rise-fall with a $\cos ^{2}$ onset envelope, delivered at $40 / \mathrm{s}$ ). The response was amplified $(\times 10,000)$, filtered $(100 \mathrm{~Hz}$ to $3 \mathrm{kHz})$, and averaged in BioSig computer software (Tucker-Davis Technologies, Alachua, FL, USA). The sound level was decreased in 5-dB steps from 99-dB SPL down to 15-dB SPL. At each sound level, 1024 responses were averaged, with stimulus polarity alternated. Response waveforms were discarded as artifact if the peak-topeak voltage exceeded $15 \mu \mathrm{V}$. Stimulus frequencies were $5.6,8,11,16,22.63,32,45.2$, and $64 \mathrm{kHz}$ for threshold testing. Threshold was determined by a single observer, who noted the lowest sound level at which a recognizable waveform was seen on a screen of tracings stacked from highest to lowest sound level. Waveforms were confirmed as auditory-evoked responses by their increasing latency and decreasing amplitude with decreasing sound intensity of the stimulus. If hearing threshold was not detected at $99 \mathrm{~dB}$, a threshold value of $100 \mathrm{~dB}$ was assigned.

\section{Histology}

Immediately after ABR test, animals were perfused with $4.0 \%$ paraformaldehyde via direct cardiac cannulation. Both petrous temporal bones were extracted, and the oval window opened to allow intralabyrinthine perfusion of fixative. After overnight fixation at $4^{\circ} \mathrm{C}$, cochleas were decalcified in $0.1 \mathrm{M}$ buffered ethylenediaminetetraacetic acid for $3-5$ days at $4^{\circ} \mathrm{C}$.

\section{Immunohistochemistry}

After decalcification, left cochleas were immersed in cryoprotection solution overnight, frozen in $30 \%$ sucrose on dry ice, and cut into $30-\mu \mathrm{m}$ sections from round window to oval window on a horizontal sliding microtome. The sections were then washed in PBS ( $\mathrm{pH} 7.4$ ) and dried onto slides for $10 \mathrm{~min}$ in a $60^{\circ} \mathrm{C}$ oven. All subsequent steps were followed by three 5-min washes with PBS and were carried out at room temperature. For Figure 3, streptavidin/horseradish peroxidase/diaminobenzidine was used. Endogenous peroxidase activity was blocked with $1 \%$ hydrogen peroxide in $0.1 \%$ Triton X-100/PBS for $30 \mathrm{~min}$. Tissue sections were incubated in 3\% normal goat serum in PBS for $1 \mathrm{~h}$ and then incubated overnight in 1:8,000 rat anti-mouse CD45 (Serotec, Oxford, UK) in a humidified chamber at room temperature. Rat antimouse CD45 monoclonal antibody used in this study was raised against purified B cells from mouse lymph nodes and labels a single band of 100-150 kD molecular weight in mouse brain extracts on Western blot (Cuadros et al. 2006). Subsequently, cochlear sections were incubated in 1:1,000 biotinylated goat anti-rat secondary antibody for $1 \mathrm{~h}$ and processed using a standard streptavidin-biotin-horseradish peroxidase reagent kit (Vector Laboratories, Burlingame, CA, USA) followed by $0.05 \% \quad 3,3^{\prime}$ diaminobenzidine tetrahydrochloride (DAB, Sigma Chemical, St. Louis, MO, USA) and $0.01 \%$ hydrogen peroxide for $5 \mathrm{~min}$, post-fixed in $0.04 \%$ osmium tetraoxide for $30 \mathrm{~s}$, and cover-slipped in glycerol.

For images and cell counts in Figure 5, rat anti-mouse CD45 and fluorescein isothiocyanate (FITC)-conjugated secondary antibodies were used in lieu of biotinylated secondary antibodies (AlexaFluor 594 goat anti-rat, 1:5,000, Molecular Probes, Eugene, OR, USA). These images were generated on a confocal microscope with a $\times 20$ objective lens (Leica TCS confocal microscope). Negative controls were performed with no primary antibody to detect background labeling. In Figures 4 and 5, endogenous green fluorescent protein whose expression was controlled by the CX3CR1 promoter in CX3CR1/green fluorescent protein mice are also shown (Jung et al. 2000).

\section{Morphometric analysis}

Decalcified right cochleas were post-fixed in osmium $\left(1 \% \mathrm{OsO} 4 \mathrm{in} \mathrm{dH}_{2} \mathrm{O}\right.$ ) for $60 \mathrm{~min}$. Cochleas were dehydrated through a series of graded ethanols and 
then placed in propylene oxide and embedded in Araldite resin. Plastic embedded cochleas were sectioned at $40 \mu \mathrm{m}$ in a horizontal plane parallel to the spiral axis of the modiolus. Each section was mounted and cover-slipped in Permount.

The cochlear duct was reconstructed and mapped in three dimensions. A specific point in each cochlear profile was chosen to define the location of the sensory epithelium. This point was identified on the projected image of the first section in the series, and subsequent sections were aligned and superimposed as a stack of images. The fixed point in each section was given a three-dimensional coordinate by a 3D computer software program (Neurolucida, Microbrightfield, Burlington, VT, USA). From these 3D coordinates, the entire cochlear duct was reconstructed, and the length of the cochlea was determined. Cochlear location was converted into frequency according to a frequency-map data described by Ehret (1983) which was in conformity with to the mathematical equation $f(\mathrm{kHz})=3.109 \times\left(10^{(100-\mathrm{d}) \times 0.0142}-0.7719\right)$, where $d=$ distance from the base.

\section{Hair cells}

A standard cytocochleogram was prepared for each ear using $\times 100$ objectives and Nomarski optics. In each section of the series, the percent of surviving outer and inner hair cells was assessed for each profile through the cochlear duct of the entire section thickness. The presence of a nucleus and stereocilia were necessary criteria to establish the presence of a hair cell. An empty Deiter cup would be evidence of a missing hair cell.

\section{Spiral ligament and spiral limbus fibrocytes}

A single observer estimated the percentage of surviving fibrocytes in every section of the series. Estimates of cell loss were performed with a $\times 40$ objective and were reported as the percent of fibrocytes remaining as compared to control ears.

\section{For counting CD45+ or CX3CR1+ cells}

Five to six cochlear sections for each mouse were viewed on a standard epifluorescence microscope with a $\times 40$ objective. The cochlea was divided into four regions (lower basal, upper basal, lower apical, and upper apical turns) and assigned the mean frequency for this region of the mouse cochlea. The presence of labeled cytoplasm and a cell nucleus were used to determine the presence of a CD45+ or CX3CR1+ /GFP cell.

\section{Statistical analysis}

The Mann-Whitney $U$ test was used for the statistical analysis of two unpaired samples. Analysis of variance
(ANOVA) was used for multiple sample statistical analysis. A probability $(p)$ value $<0.05$ was considered significant.

\section{RESULTS}

Absence of CX3CR1 exacerbates hearing loss and outer hair cell damage after kanamycin

Figure 1 shows ABR thresholds in saline-injected controls where hearing thresholds were similar in all three genotypes (Fig. 1A). After kanamycin, ABR thresholds were worse in CX3CR1 knockout mice compared to heterozygous and wild-type littermates (Fig. 1B). In the CX3CR1 heterozygous and wild-type mice, ABR thresholds in the lower frequencies remained normal, and threshold elevation was detected at $16 \mathrm{kHz}$ and higher frequencies. In

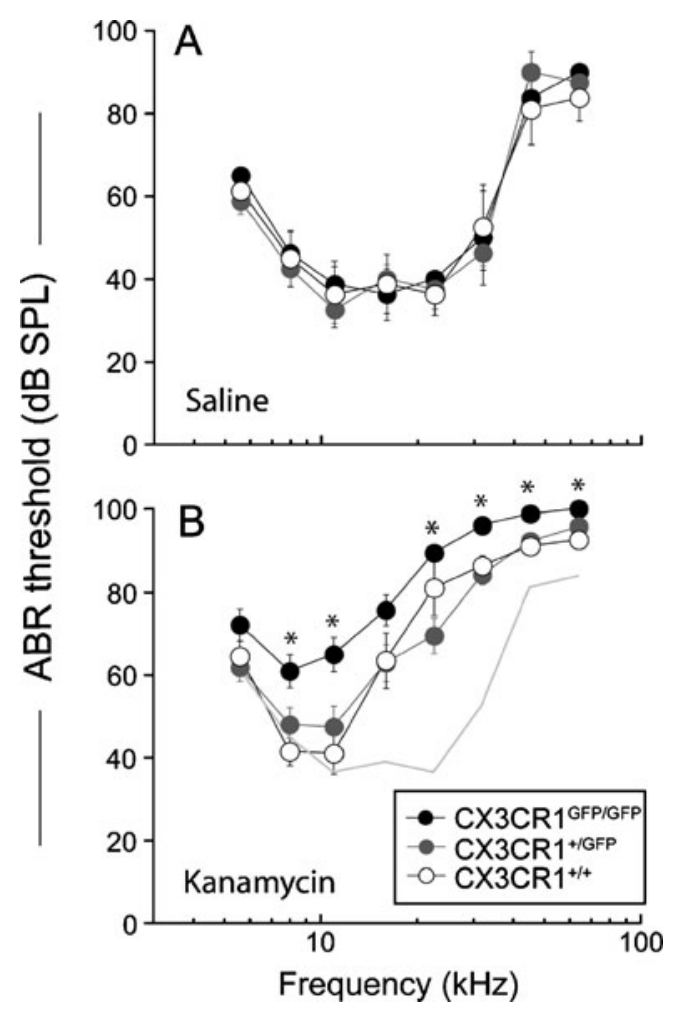

FIG. 1. Auditory brainstem response (ABR) thresholds after kanamycin in wild-type, CX3CR1 heterozygous, and CX3CR1 knockout mice. A Mice from three Cx3CR1 genotypes demonstrated similar hearing thresholds after saline control treatment $(n=4$ mice per group). B After kanamycin, ABR thresholds were elevated at all frequencies above $16 \mathrm{kHz}$ in all groups. The solid gray line represents the average threshold in all three genotypes injected with saline shown in A. In CX3CR1 knockout mice, ABR thresholds were elevated in the low frequencies as well as in the high frequencies. ABR thresholds were significantly different in knockout mice when compared to wild-type mice at $8,11,22.63,32,45.2$, and $64 \mathrm{kHz}$ (asterisk indicates probability of ANOVA is less than $0.05 ; \mathrm{WT}, n=10$; $\mathrm{HET}, n=22 ; \mathrm{KO}, n=22$ ). Icons represent the mean, and error bars represent standard error of the mean. 
contrast, ABR thresholds in CX3CR1 knockout mice were elevated across all. The differences in ABR thresholds were statistically significant at 8, 11, $22.63,32,45.2$, and $64 \mathrm{kHz}(p<0.05$, ANOVA) when we compared knockouts to heterozygotes and wild-types.

Figure 2A demonstrates average cytocochleograms for wild-type, heterozygous, and knockout mice in saline controls or kanamycin-treated ears. These cytocochleograms represent the percent of surviving outer hair cells on the $y$-axis graphed against frequency on the $x$-axis. Saline-injected mice exhibited a small percentage of degenerated outer hair cells in the high frequency region, which was consistent across all three genotypes. In the kanamycin-treated animals, outer hair cell damage was seen extending from the high frequency region to the mid-frequency region. In all three genotypes, a large proportion of mice demonstrated degeneration of outer hair cells in the basal turn of the cochlea $(20 \mathrm{kHz}$ and higher frequency region). However, only the CX3CR1 knockout mice demonstrated outer hair cell loss in the mid to low frequency region of the cochlear duct. Outer hair cell loss extended further apically in knockout mice than in heterozygous or wild-type mice, consistent with $\mathrm{ABR}$ thresholds demonstrating greater losses in the low frequency region. Direct comparison of groups demonstrates statistically significant differences in outer hair cell survival at locations correspond-

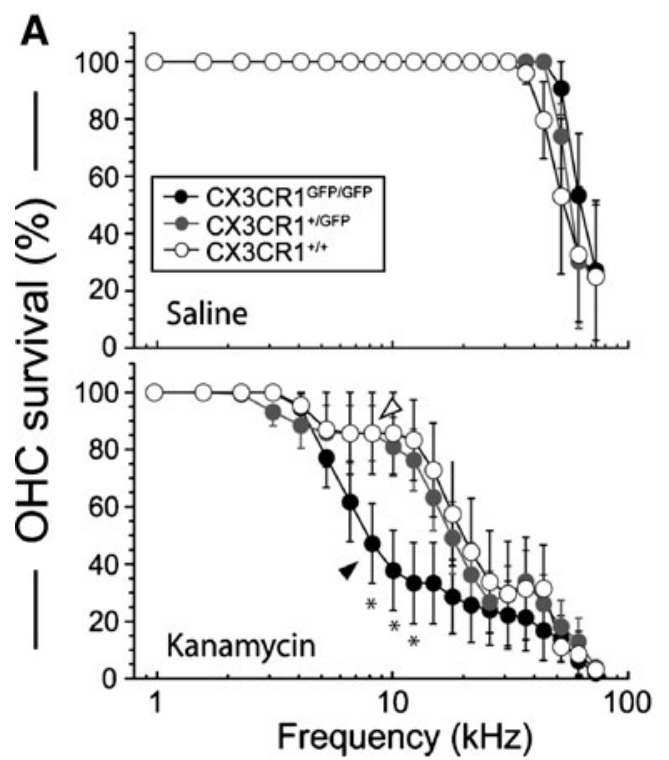

FIG. 2. CX3CR1 knockout mice suffered greater hair cell damage than wild-type mice after kanamycin. A Cytocochleograms demonstrate that outer hair cell loss was minimal among the saline-injected groups. In kanamycin-exposed animals, CX3CR1 knockout mice suffered greater hair cell loss than heterozygous mice or wild-type mice from 5 to $16 \mathrm{kHz}$ region. The differences were significant when we compared the average hair cell survival for heterozygous and wild-type mice with the average hair cell survival in knockout mice at 8,10 , and $12 \mathrm{kHz}$ (asterisk indicates $p<0.05$ in analysis of ing to 8,10 , and $12 \mathrm{kHz}$ (marked with an asterisk in Fig. 2, $p<0.05$, ANOVA).

Figure 2B shows a light microscopic image of a representative section of the organ of Corti in the low frequency region of a wild-type mouse cochlea after kanamycin. All outer and inner hair cells remained in the low frequency region. Figure 2C shows the organ of Corti in the same region of a representative CX3CR1 knockout mouse cochlea after kanamycin. All outer hair cells were absent, while the inner hair cells, Deiters cells, and pillar cells remained. After kanamycin, some loss of inner hair cells in the high frequency basal region of the cochlea occurred in all three genotypes with no significant difference between groups. The spiral ligament was not damaged after ototoxic injury when compared to the control group. The spiral limbus was also not significantly altered in any of the groups.

Inflammatory cells accumulate in the cochlea after kanamycin

Wild-type mouse cochleas were harvested 15 days after twice daily kanamycin, and frozen sections of these mouse cochleas were immunostained with CD45 demonstrating the presence of mononuclear phagocytes in the lateral wall and adjacent to the dendrites of the eighth nerve. Figure $3 \mathrm{~A}$ and $\mathrm{B}$ demonstrate CD45 labeling in the upper basal turn in control and kanamycin-treated ears. In control mice, CD45

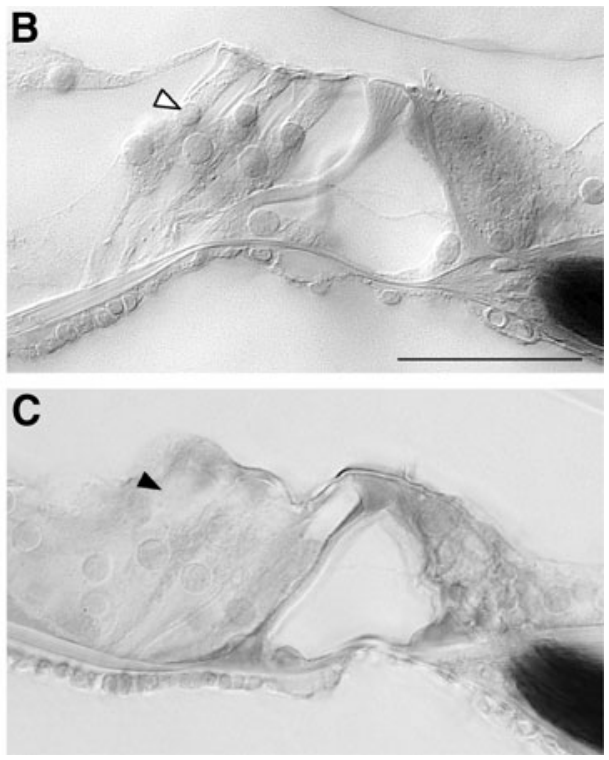

variance; $n=4$ mice in each of the saline-injected groups. In each kanamycin exposed group: $\mathrm{WT}, n=7 ; \mathrm{HET}, n=14 ; \mathrm{KO}, n=12)$. Icons show means, and error bars show SD. B A representative section of a wild-type mouse cochlea demonstrates preservation of outer hair cells in the apical turn (white arrowhead demonstrates outer hair cells that are intact in this region). C CX3CR1 knockout mice demonstrated a definitive loss of outer hair cells in the $8-12-\mathrm{kHz}$ region after kanamycin (black arrowhead). Scale bar in B $(25 \mu \mathrm{m})$ applies to $\mathbf{B}$ and $\mathbf{C}$. 

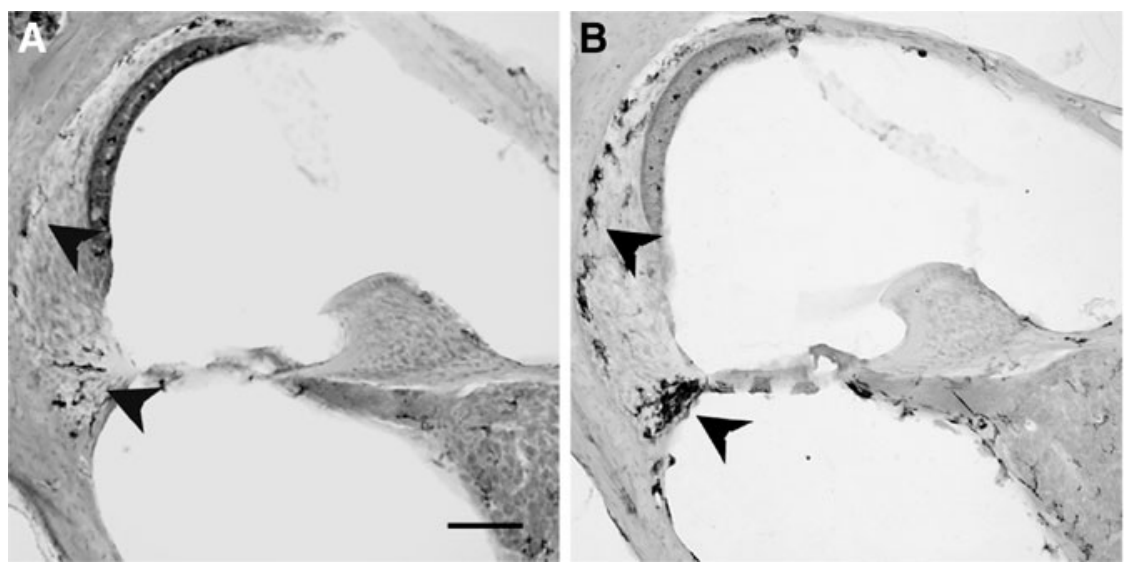

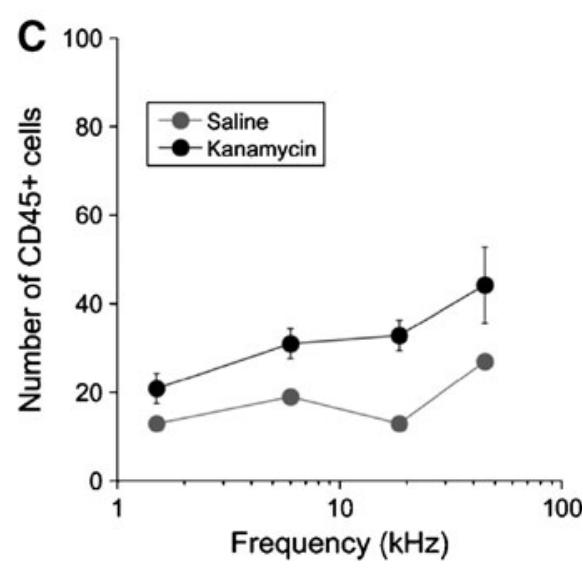

increase in leukocytes from base to apex, concentrated particularly in the high frequency region. The upper basal turn of the cochlea is shown in both panels $\mathbf{A}$ and $\mathbf{B}$. Scale bar in $\mathbf{A}(100 \mu \mathrm{m})$ applies to $\mathbf{A}$ and $\mathbf{B}$ ( $n=4$ mice in saline-injected and four mice in kanamycininjected wild-type groups). Icons in $\mathbf{C}$ represent the mean, and error bars represent standard error of the mean. positive cells were sparsely distributed in the lower portion of the spiral ligament and near the dendrites of the spiral ganglion cells (Fig. 3A). After kanamycin treatment, these cells increased in number, particularly in the lower portion of the spiral ligament (Fig. 3B). Mononuclear phagocytes were also seen in the scala tympani and the spiral ganglion. An increase in the total number of cochlear inflammatory cells was observed throughout the entire length of the cochlear duct as shown in the graph in Figure 3C, and they were concentrated in the high frequency region.

After kanamycin, cochlear macrophages are more abundant in fractalkine receptor knockout mice than in wild-type mice

Confocal images of CX3CR1 heterozygous (4A\&B) and knockout (4D\&E) mouse cochleas after kanamycin treatment are shown in Figure 4. Green fluorescent protein was expressed endogenously in monocytes, macrophages, and microglia here (Jung et al. 2000). Macrophages were more abundant in CX3CR1 knockout mice after kanamycin. Macrophages were concentrated in the lower portion of the spiral ligament in both genotypes. The morphology of cochlear monocytes/ macrophages was similar in both CX3CR1 heterozygous and knockout mice. The distribution of macrophages favored the high frequency region in both genotypes, but there were clearly more cells in CX3CR1 knockout mice (Fig. 4E). The difference in number of macrophages in knockout versus heterozygous mice was statistically significant (noted by asterisks, $n=5$ mice per group, $p<0.05$, Mann-Whitney $U$ test).
Mice with CX3CR1-deficient marrow demonstrate exacerbation of hair cell loss after kanamycin

In order to isolate the effect of CX3CR1 deletion and eliminate possible effects of CX3CR1 in development, we transplanted donor bone marrow from CX3CR1 knockout mice into irradiated wild-type recipient mice. Because irradiation and transplantation added another 4 weeks to the experimental protocol, our test mice were $12-16$ weeks of age at the time of final analysis. To minimize the effect of age-related high frequency hearing loss known to be present in inbred C57B16 mice, we used a similar but slightly altered version of this mouse. B6.CAST mice possess the allele from outbred Mus musculus castaneus in the locus of cadherin 23, one of the genes responsible for agerelated hearing loss in C57BL6 mice. B6.CAST mice possess more than $99 \%$ of the C57BL6 genome but do not exhibit high frequency age-related hearing loss typically seen in this inbred strain (Noben-Trauth et al. 2003).

In this experiment, native bone marrow was ablated by lethal doses of radiation, and bone marrow was replaced by transplantation of either wild-type, CX3CR1 heterozygous, or CX3CR1 knockout bone marrow. Two weeks after transplantation, mice had accepted their transplanted bone marrow and were subsequently exposed to kanamycin twice daily for 15 days (Sato et al. 2008). We tested three kanamycin doses $(700,900$, and $1,100 \mathrm{mg} / \mathrm{kg}$ ) in wild-type B6.CAST mice and in bone marrow chimeras (data not shown). Bone marrow chimeras were found to be more susceptible to kanamycin than control B6.CAST mice; thus, doses of kanamycin were adjusted for experiments in the bone marrow chimeras. 

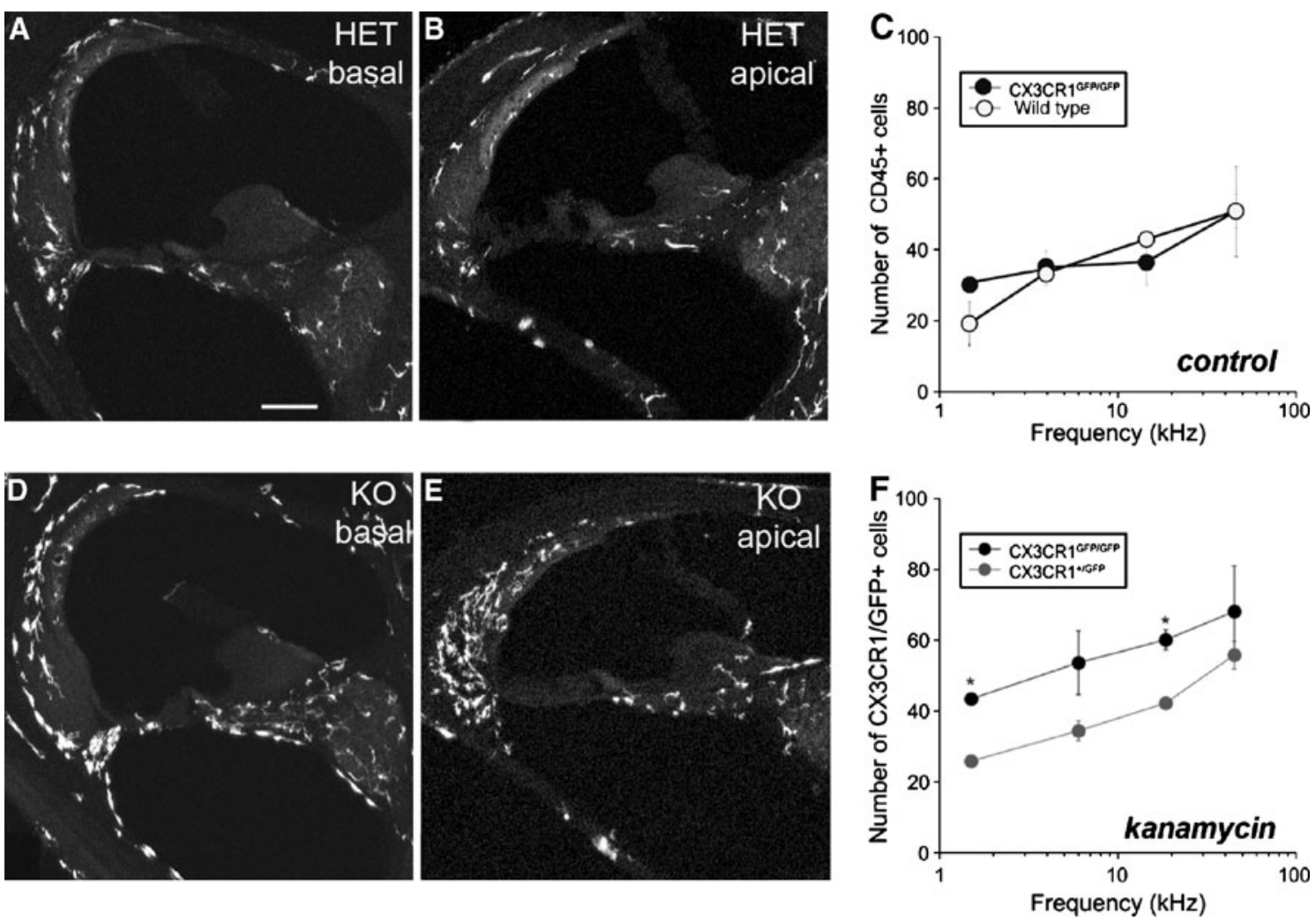

FIG. 4. Absence of CX3CR1 results in increased macrophage accumulation after kanamycin exposure. Representative sections from a CX3CR1 heterozygous mouse (A: base and $\mathbf{B}$ : apex) were compared to sections from a CX3CR1 knockout mouse (D: base and E: apex) after kanamycin treatment. Images were photographed on a Leica confocal microscope with the $\times 20$ objective lens. Macrophages were identified by their endogenous green fluorescent protein expression, driven by the CX3CR1 promoter, labeling all cells that normally express CX3CR1 (monocytes, macrophages, and

We performed ABR thresholds, cytocochleograms, and cochlear macrophage cell counts after 15 days of twice daily kanamycin in seven to eight animal subjects of each group. The data for individual subjects are shown in Figure 5 separated by genotype of the donor marrow. In the bottom row of this figure, representative sections are shown illustrating cochlear macrophages from each group. There was no significant difference between the three genotypes when averages of ABR thresholds, number of recruited monocytes, or hair cell counts were compared $(p>0.05$, ANOVA). However, large variations were observed within each group. In some subjects, a substantial influx of cochlear mononuclear phagocytes was present particularly in the spiral ligament, whereas in others, the number of recruited phagocytes was modest.

Compared to non-irradiated subjects, the bone marrow chimeras demonstrated considerably more inflammatory cells in the inner ear after kanamycin. Given a high degree of variability in hair cell loss and microglia). Cell counts showed that CX3CR1 knockout mice elicit an increased number of macrophages after kanamycin at all frequencies (F) when compared with CX3CR1 heterozygous mice. Panel C demonstrates that numbers of cochlear mononuclear phagocytes at baseline were similar between wild-type and knockout mice. ( $N=5$ $\mathrm{KO}, \mathrm{N}=5 \mathrm{WT}$ mice used for cell counts). The icons represent means and standard error of the mean. In F, differences in macrophage cell number were statistically significant at the upper basal and upper apical turns ( $N=5 \mathrm{KO}$ and $4 \mathrm{HET}$ mice).

numbers of recruited leukocytes within each group, we questioned whether we might detect an effect of macrophage number on hair cell survival in any of the experimental groups. We calculated the total percent survival of outer hair cells in each cochlea by averaging the cytocochleogram data across all frequencies and performed a linear regression analysis to determine the effect of macrophage number on hair cell survival. Figure 6 demonstrates this linear regression analysis correlating outer hair cell survival to number of cochlear macrophages using each animal subject from each of the three experimental groups as a data point. A significant correlation was found between numbers of cochlear macrophages and loss of outer hair cells in CX3CR1 knockout chimeras $\left(R^{2}=0.72, p=0.007\right)$. However, this correlation was not present in either wild-type or heterozygote chimeras. Only in CX3CR1 knockout chimeras, more cochlear macrophages correlated with worse hair cell outcome. These results suggest that CX3CR1 null leukocytes confer increased susceptibility to 

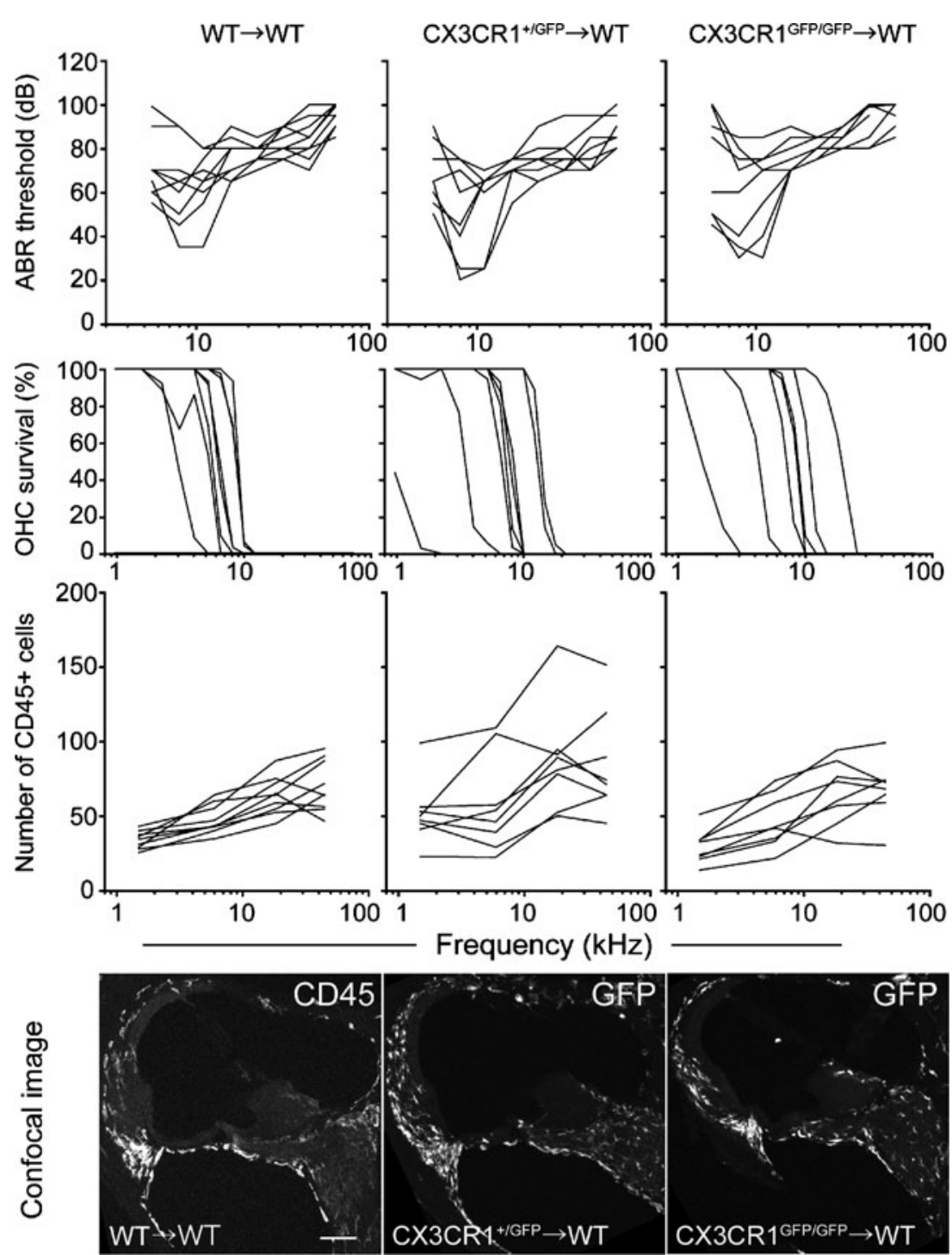

FIG. 5. Auditory brainstem response (ABR) thresholds, cochlear macrophage number, and outer hair cell survival of wild-type, heterozygous, and knockout chimeras. There were large variations within each genotype with respect to the ABR thresholds, leukocyte recruitment, and outer hair cell survival. There was no significant difference in the average $A B R$ thresholds, number of cochlear leukocytes, or outer hair cell survival after kanamycin treatment. The final row of this figure demonstrates representative sections of the cochlea after immunostaining for CD45 in each of the three genotypes. Abundant leukocyte recruitment was observed in all three groups of bone marrow chimeras. kanamycin-induced hair cell loss and negatively impact hair cell survival. CX3CR1 positive macrophages correlated with no change in the susceptibility of hair cells to ototoxicity. We postulate that macrophages that lack CX3CR1 have lost important regulatory cues that limit damage incurred by inflammatory cells when recruited to a site of injury.

\section{DISCUSSION}

In this study, we have shown that mononuclear phagocytes accumulate in the lateral wall of the cochlea after aminoglycoside injury and that CX3CR1 appears to influence the outcome of hearing loss by regulating the effector function of these cells after kanamycin. Following ototoxic injury, the lateral wall and the surfaces of the scala tympani and vestibuli are populated with monocytes and macrophages. Despite the notable presence of these inflammatory cells, non-sensory cells in these areas do not demonstrate signs of damage. Phagocytic cells from circulation may enter the ear via the lateral wall because it represents the most permeable barrier for cells to traverse from blood to labyrinth. Precisely what cues are present in the inner ear that encourage the migration and arrest of mononuclear phagocytes in the inner ear are not yet known although CCL2 and CCR2, which play an important role in 


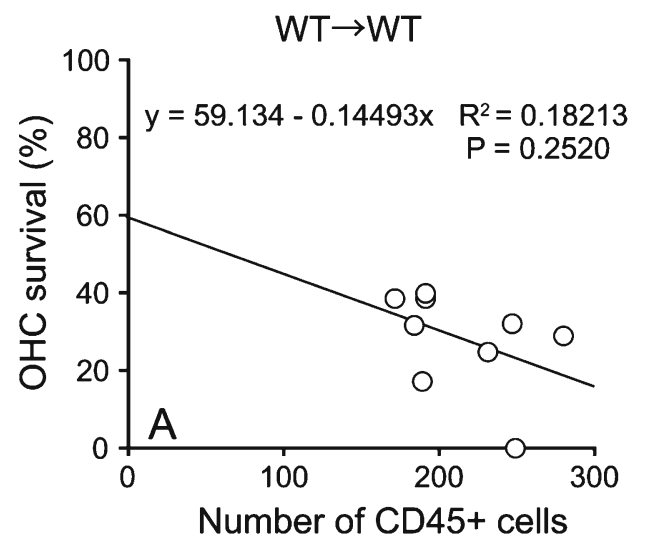

FIG. 6. Linear regression demonstrates a positive correlation between macrophage number and hair cell loss in bone marrow chimeras that received CX3CR1 knockout marrow. Linear regression analysis demonstrates the relationship between the number of cochlear macrophages and outer hair cell survival. In this experiment, a statistically significant positive correlation between macrophage number and hair cell loss in

monocyte migration in brain and spinal cord, appear not to be critical cues for monocyte migration in the ear (Sautter et al. 2006).

Hair cell death due to aminoglycoside ototoxicity has been considered largely an apoptotic process and was not expected to be accompanied by an inflammatory response; thus, the abundance of cochlear inflammatory cells after kanamycin was not anticipated (Matsui et al. 2003; Nakagawa et al. 2003; Lee et al. 2004; Tabuchi et al. 2007; Goodyear et al. 2008; Oesterle et al. 2008; Taleb et al. 2008; Taylor et al. 2008). In this paper, we question whether the presence of inflammatory cells results in exacerbation of ototoxic injury or if they play a neutral role in phagocytosis of degenerated cells or even a beneficial role in repair of the sensory epithelium. In avian ototoxicity, authors have considered the possibility that inflammatory cells recruited by hair cell injury could contribute to regeneration (Warchol 1997, 1999; Bhave et al. 1998). In a recent paper exploring rat hair cell death after amikacin, macrophages were identified as an important limiting step in the process of supporting cell transdifferentiation into hair cells (Ladrech et al. 2007). While the contributions of macrophage-derived cytokines and growth factors in regeneration are currently unknown, clearance of hair cell debris and cytokine production that regulates further inflammation are potentially important roles for these cochlear leukocytes that might foster repair or even regeneration. Macrophages as professional phagocytes could be important in maintaining the separation between endolymph and perilymph by clearing ruptured cells in the reticular lamina and might therefore protect residual hearing in nondamaged, low frequency regions of the inner ear. In addition, tissue macrophages are known to release cytokines that either limit or elicit further inflammation, which could also impact the final outcome of ototoxicity.

In our prior work, we characterized CX3CR1 in cochlear macrophages after acoustic trauma (Hirose et al. 2005; Sato et al. 2008). The function of leukocytes in the injured mammalian inner ear and the cues that regulate their activation are poorly understood at this time. In the central nervous system, CX3CL1 and CX3CR1 have been shown to modulate microglial activation and neurotoxicity in various conditions of brain injury (Harrison et al. 1998; Limatola et al. 2005a; Cardona et al. 2006). Our current study confirms high frequency hearing loss and degeneration of high frequency outer hair cells in mammals exposed to kanamycin (Henry et al. 1981; Chen and Saunders 1983; Wu et al. 2001; Jiang et al. 2006; Taleb et al. 2009). Additionally, we demonstrate that CX3CR1 knockout mice suffer more extensive hair cell loss and hearing loss when compared to CX3CR1-expressing mice. After kanamycin, hearing loss in the high frequencies reaches the limits of testing in all genotypes. Notably, low frequency hearing is preserved in control and heterozygous mice but is severely impaired in CX3CR1 knockout mice. Additionally, there are more macrophages present overall and macrophages extend higher into the apical turn in CX3CR1 knockout mice compared to wild-type or heterozygous mice. CX3CR1 may be an important signal to limit the migration of monocytes from circulation into peripheral tissues, and monocytes lacking cues from CX3CR1 signaling may propagate hair cell injury beyond the primary lesion caused by kanamycin.

In order to isolate the effect of CX3CR1 deletion to mature hematopoietic cells, we generated CX3CR1- 
deficient bone marrow chimeras. In wild-type mice transplanted with CX3CR1-knockout bone marrow, we found a strong correlation between number of macrophages and degree of hair cell loss. In contrast, this correlation between number of macrophages and severity of hair cell loss was not present in mice that received either wild-type or heterozygous bone marrow. Based on our findings, we propose that in bone marrow chimeras, CX3CR1 suppresses deleterious effects of cochlear macrophages by regulating their activity without effectively regulating their number. In bone marrow chimeras, we did not detect an effect of CX3CR1 on monocyte accumulation likely due to the prevailing effect of radiation on leukocyte permeability.

In a prior study comparing the technique of bone marrow chimeras to parabiosis, where two animals are sutured together to achieve complete mixing of blood, authors showed that donor brain microglia are present in recipients after radiation and bone marrow transplantation but are not observed in recipient parabionts (Ajami et al. 2007). This difference is attributed to radiation-induced disruption of the blood-brain barrier allowing entry of donor bone marrow cells into the brain and their differentiation into microglia. It is possible that radiation, used to ablate native bone marrow, resulted in increased leukocyte permeability at the blood-labyrinth barrier in our chimeras. While the knockout mice demonstrated that CX3CR1 is important in regulating monocyte trafficking, this effect of CX3CR1 was obscured by the effect of radiation in the bone marrow chimeras. In the wild-type and heterozygous bone marrow chimeras, hair cell outcome was indifferent to macrophage number, whereas in knockouts, hair cell outcome was better when there were fewer macrophages and worse with larger numbers of macrophages. It appears that in wild-type and heterozygous CX3CR1 bone marrow recipients, hair cell outcome is determined by factors independent of leukocyte migration whereas in CX3CR1 knockout recipients, macrophage number could play a role in outcome.

How expression of a receptor on macrophages in the spiral ligament affect sensory cells in the organ of Corti is an important question that remains unanswered at this time. Cochlear macrophages were rarely seen in the sensory epithelium, and we did not observe macrophages in the scala media. In a prior study on macrophages in amikacin ototoxicity, the authors reported numerous macrophages in the organ of Corti (Ladrech et al. 2007). However, because these specimens were prepared as whole mounts of the sensory epithelium, we believe that these leukocytes might have been lying below the basilar membrane in the scala tympani and not at the hair cell level, as we have observed cells in this location. We hypothesize that the high potassium environment of the endolymph may be incompatible with survival of peripheral blood mononuclear cells and that interactions between mononuclear phagocytes and hair cells are brief and might involve extension of processes without migration of the cell body. While direct physical interaction between cochlear macrophages and hair cells appeared rare, there are various scenarios in the central nervous system where macrophages and microglia modulate damage in neurodegeneration via numerous mechanisms, some of which involve signaling through CX3CR1 (Cotter et al. 2002; Nguyen et al. 2004; Cardona et al. 2006; Neumann et al. 2006; Denes et al. 2008) .

Cochlear macrophages are observed in stable numbers in the stria vascularis after kanamycin. While macrophages in the stria have been implicated as a source of hearing loss in a mouse model of Pendred's syndrome, this does not appear to be the case after ototoxic injury (Jabba et al. 2006). Earlier work has shown loss of marginal cell volume in the stria vascularis after systemic gentamicin administration in guinea pigs (Forge and Fradis 1985). These changes in the strial marginal cells cannot be readily explained by the presence of macrophages in this area. Further studies involving measurement of the endocochlear potential could potentially link CX3CR1 expression in spiral ligament macrophages and threshold shift after kanamycin-induced ototoxicity.

Cochlear macrophages routinely migrated from the lateral wall into the scala tympani and were observed in regions directly in contact with the basilar membrane. One might speculate that CX3CL1, the ligand for CX3CR1, arises from damaged hair cells and regulates entry of mononuclear phagocytes to the inner ear. However, it is clear that CX3CR1 is not necessary for monocyte migration, as the knockout mice demonstrated robust macrophage entry into the inner ear. In fact, it appears more likely that CX3CR1 signaling may be necessary to limit or refine the entry of leukocytes into the inner ear, as CX3CR1 null mice demonstrated greater numbers of recruited phagocytes after kanamycin exposure when compared to wild-type mice. It is possible that production of the inflammatory cytokines, IL-1 beta, TNF alpha, and IL-6, which are typically released by activated macrophages, plays a role in exacerbating kanamycin injury. In prior studies of cisplatinum ototoxicity, expression of these inflammatory cytokines appears to be an important mediator of hair cell damage (So et al. 2008). It is possible that CX3CR1 null macrophages in the inner ear constitutively express certain inflammatory cytokines as a result of lack of CX3CR1-mediated inhibition, although this hypothesis has not been tested. 
In summary, our findings suggest that fractalkine receptor, CX3CR1, may play a protective role in the cochlea and that disruption of CX3CR1 potentiates leukocyte entry in the ear, exacerbates hearing loss, and hair cell damage after kanamycin in CX3CR1 knockout mice. The mechanism by which CX3CL1, the exclusive ligand for fractalkine receptor, could exert a protective effect is not clear. It appears that CX3CR1 is expressed exclusively in hematopoietically derived cells in the inner ear, and transplantation of these cells from CX3CR1 knockout mice to wild-type mice results in worse hair cell outcomes with increasing numbers of monocytes. Which cells in the cochlea express or secrete CX3CL1 is unknown at this time. We conclude that CX3CR1 null cochlear macrophages lack the normal suppressive cues that are properties of CX3CR1 signaling and could play a role in exacerbating ototoxicity in the face of aminoglycoside injury.

\section{ACKNOWLEDGEMENTS}

We thank Grahame Kidd for his assistance with confocal microscopy and Astrid Cardona and Liping Liu for technical advice, and Mark Warchol, Tim Hullar, and Song Zhe Li for helpful discussion of this manuscript. This work was funded by NIH grant K08DC005761, the Royal National Institute for the Deaf, and a grant from the Triple $\mathrm{T}$ Foundation and by Nancy Lerner Fisher.

\section{REFERENCES}

Ajami B, Bennett JL, Krieger C, Tetzlaff W, Rossi FM (2007) Local self-renewal can sustain CNS microglia maintenance and function throughout adult life. Nat Neurosci 10:1538-1543

Bhave SA, Oesterle EC, Coltrera MD (1998) Macrophage and microglia-like cells in the avian inner ear. J Comp Neurol 398:241-256

Browning GG, Northrop R, CorTese R (1982) Selective destruction of the outer hair cells in the chinchilla. Clin Otolaryngol Allied Sci 7:3-9

Cardona Ae, Pioro EP, Sasse Me, Kostenko V, Cardona SM, Dijkstra IM, Huang D, Kidd G, Dombrowski S, Dutta R, Lee JC, Cook DN, Jung S, Lira SA, Littman DR, Ransohoff RM (2006) Control of microglial neurotoxicity by the fractalkine receptor. Nat Neurosci 9:917-924

Chen CS, Saunders JC (1983) The sensitive period for ototoxicity of kanamycin in mice: morphological evidence. Arch Otorhinolaryngol 238:217-223

Cotter R, Williams C, Rian L, Erichsen D, Lopez A, Peng H, Zheng J (2002) Fractalkine (CX3CL1) and brain inflammation: implications for HIV-1-associated dementia. J Neurovirology 8:585-598

Cudaroos MA, Santos AM, Martin-Oliva D, Calvente R, Tassi M, Marin-Teva JL, Navascues J (2006) Specific immunolabeling of brain macrophages and microglial cells in the developing and mature chick central nervous system. J Histochem Cytochem 54:727-738

Denes A, Ferenczi S, Halasz J, Kornyei Z, Kovacs KJ (2008) Role of CX3CR1 (fractalkine receptor) in brain damage and inflamma- tion induced by focal cerebral ischemia in mouse. J Cereb Blood Flow Metab 28:1707-1721

EHREт G (1983) Peripheral anatomy and physiology II. In: Willott J (ed) The auditory psychobiology of the mouse. Springfield, IL: Charles C. Thomas, p 169-200

Forge A, Fradis M (1985) Structural abnormalities in the stria vascularis following chronic gentamicin treatment. Hear Res 20:233-244

Fredelius L, Rask-Andersen H (1990) The role of macrophages in the disposal of degeneration products within the organ of corti after acoustic overstimulation. Acta Otolaryngol 109:76-82

Goodyear RJ, Gale Je, Ranatunga KM, Kros CJ, Richardson GP (2008) Aminoglycoside-induced phosphatidylserine externalization in sensory hair cells is regionally restricted, rapid, and reversible. J Neurosci 28:9939-9952

Harrison JK, Jiang Y, Chen S, Xia Y, Maciejewski D, McNamara RK, Streit WJ, Salafranca MN, Adhikari S, Thompson DA, Botti P, Bacon KB, Feng L (1998) Role for neuronally derived fractalkine in mediating interactions between neurons and CX3CR1-expressing microglia. Proc Natl Acad Sci USA 95:10896-10901

Henry KR, Chole RA, McGinn MD, Frush DP (1981) Increased ototoxicity in both young and old mice. Arch Otolaryngol 107:92-95

Hirose K, Discolo CM, Keasler JR, Ransohoff R (2005) Mononuclear phagocytes migrate into the murine cochlea after acoustic trauma. J Comp Neurol 489:180-194

Jabba SV, Oelke A, Singh R, Maganti RJ, Fleming S, Wall SM, Everett LA, Green ED, Wangemann P (2006) Macrophage invasion contributes to degeneration of stria vascularis in Pendred syndrome mouse model. BMC Med 4:37

Jiang H, Sha SH, Forge A, Schacht J (2006) Caspase-independent pathways of hair cell death induced by kanamycin in vivo. Cell Death Differ 13:20-30

Jung S, Aliberti J, Graemmel P, Sunshine MJ, Kreutzberg GW, Sher A, Littman DR (2000) Analysis of fractalkine receptor CX(3)CR1 function by targeted deletion and green fluorescent protein reporter gene insertion. Mol Cell Biol 20:4106-4114

Ladrech S, Wang J, Simonneau L, Puel JL, Lenoir M (2007) Macrophage contribution to the response of the rat organ of Corti to amikacin. J Neurosci Res 85:1970-1979

Lauro C, Di Angelantonio S, Cipriani R, Sobrero F, Antonilli L, Brusadin V, Ragozzino D, Limatola C (2008) Activity of adenosine receptors type 1 Is required for CX3CL1-mediated neuroprotection and neuromodulation in hippocampal neurons. J Immunol 180:7590-7596

Lee JE, Nakagawa T, Kim TS, Iguchi F, Endo T, Kita T, Murai N, Natto Y, LeE SH, Ito J (2004) Signaling pathway for apoptosis of vestibular hair cells of mice due to aminoglycosides. Acta Otolaryngol Suppl, pp 69-74

Limatola C, Lauro C, Catalano M, Ciotti M, Bertollini C, DiAngelantonio S, Ragozzino D, Eusebi F (2005a) Chemokine CX3CL1 protects rat hippocampal neurons against glutamatemediated excitotoxicity. J Neurosci 166:19-28

Limatola C, lauro C, Catalano M, Ciotti MT, Bertollini C, Di Angelantonio S, Ragozzino D, Eusebi F (2005b) Chemokine CX3CL1 protects rat hippocampal neurons against glutamatemediated excitotoxicity. J Neuroimmunol 166:19-28

Matsui Ji, Haque A, Huss D, Messana EP, Alosi JA, Roberson DW, Cotanche DA, Dickman JD, Warchol ME (2003) Caspase inhibitors promote vestibular hair cell survival and function after aminoglycoside treatment in vivo. J Neurosci 23:6111-6122

Nakagawa T, Kim TS, Murai N, Endo T, Iguchi F, Tateva I, Yamamoto N, Naito Y, Ito J (2003) A novel technique for inducing local inner ear damage. Hear Res 176:122-127

Neumann J, Gunzer M, Gutzeit HO, Ullrich O, Reymann KG, Dinkei K (2006) Microglia provide neuroprotection after ischemia. FASEB J 20:714-716 
Nguyen MD, D'Aigle T, Gowing G, Julien JP, Rivest S (2004) Exacerbation of motor neuron disease by chronic stimulation of innate immunity in a mouse model of amyotrophic lateral sclerosis. J Neurosci 24:1340-1349

Noben-Trauth K, Zheng QY, Johnson KR (2003) Association of cadherin 23 with polygenic inheritance and genetic modification of sensorineural hearing loss. Nat Genet 35:21-23

Oesterle EC, Campbell S, Taylor RR, Forge A, Hume CR (2008) Sox2 and JAGGED1 expression in normal and drug-damaged adult mouse inner ear. J Assoc Res Otolaryngol 9:65-89

Sato E, Shick HE, Ransohoff RM, Hirose K (2008) Repopulation of cochlear macrophages in murine hematopoietic progenitor cell chimeras: the role of CX3CR1. J Comp Neurol 506:930-942

Sautter NB, Shick EH, Ransohoff RM, Charo IF, Hirose K (2006) $\mathrm{CC}$ chemokine receptor 2 is protective against noise-induced hair cell death: studies in CX3CR1(+/GFP) mice. J Assoc Res Otolaryngol 7:361-372

So H, Кim H, Кim Y, Кim E, Pae HO, Chung HT, Кim HJ, Kwon KB, Lee KM, Lee HY, Moon SK, Park R (2008) Evidence that cisplatininduced auditory damage is attenuated by downregulation of pro-inflammatory cytokines via Nrf2/HO-1. J Assoc Res Otolaryngol 9:290-306
Tabuchi K, Pak K, Chavez E, Ryan AF (2007) Role of inhibitor of apoptosis protein in gentamicin-induced cochlear hair cell damage. Neuroscience 149:213-222

Taleb M, Brandon CS, Lee FS, Lomax Mi, Dillmann WH, Cunningham LL (2008) Hsp70 inhibits aminoglycoside-induced hair cell death and is necessary for the protective effect of heat shock. J Assoc Res Otolaryngol 9:277-289

Taleb M, Brandon CS, Lee FS, Harris KC, Dillmann WH, Cunningham LL (2009) Hsp70 inhibits aminoglycoside-induced hearing loss and cochlear hair cell death. Cell Stress Chaperones 14:427-437

Taylor RR, Nevill G, Forge A (2008) Rapid hair cell loss: a mouse model for cochlear lesions. J Assoc Res Otolaryngol 9:44-64

WARCHOL ME (1997) Macrophage activity in organ cultures of the avian cochlea: demonstration of a resident population and recruitment to sites of hair cell lesions. J Neurobiol 33:724-734

WARCHOL ME (1999) Immune cytokines and dexamethasone influence sensory regeneration in the avian vestibular periphery. J Neurocytol 28:889-900

Wu WJ, Sha SH, Mclaren JD, Kawamoto K, Raphael Y, Schacht J (2001) Aminoglycoside ototoxicity in adult CBA, C57BL and BALB mice and the Sprague-Dawley rat. Hear Res 158:165-178 